
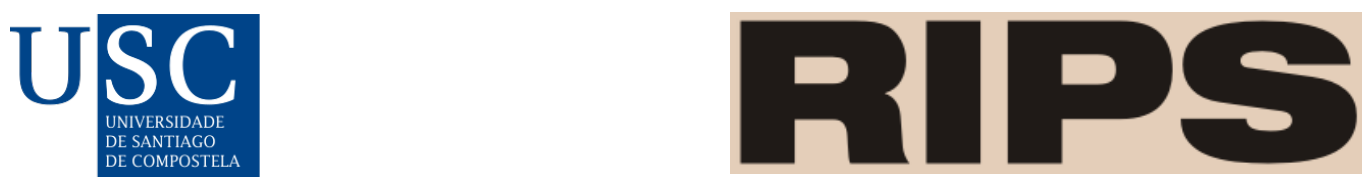

Revista

de Investigaciones

Políticas y Sociológicas

RIPS: Revista de Investigaciones Políticas y Sociológicas, 20(2), 2021. ISSN-e: 2255-5986

https://doi.org/10.15304/rips.20.2.7840

Artículos

\title{
Organización partidista y rendimiento electoral. Una aproximación al caso de la derecha radical en España
} Party organization and electoral performance. An approach to the case of the radical right in Spain

\author{
Pablo Ortiz Barquero ${ }^{1, a}$, Andrés Benítez Espinosa ${ }^{2, b}$, Alba María Aragón Morales ${ }^{3, c}$ \\ ${ }^{1}$ Universidad Pablo de Olavide, Sevilla, España \\ ${ }^{2}$ Universidad Nacional de Educación a Distancia, Madrid, España \\ ${ }^{3}$ Universidad Pablo de Olavide, Sevilla, España \\ a portbar@upo.es b andresbenitez@poli.uned.es c amaramor@upo.es
}

\section{Resumen}

En las últimas décadas, el creciente protagonismo de la familia de partidos de derecha radical en Europa ha recabado una considerable atención académica, siendo uno de los principales objetivos el estudiar su rendimiento electoral. En este sentido, la literatura ha otorgado un papel preeminente a los factores de la demanda; de forma paralela, los factores de la oferta -que son aquellos que hacen referencia a las características de los propios partidos- han recibido mucha menos atención. No obstante, existen motivos para pensar que, dadas las limitaciones de las explicaciones centradas en la demanda, la agenda de investigación sobre la derecha radical necesita de una suerte de "giro internalista". o lo que es lo mismo: una mirada más rigurosa y sistematizada sobre los factores de la oferta (y en especial, sobre aquellos relacionados con la organización partidista). El objetivo de esta investigación es examinar el impacto de la dimensión organizativa en el rendimiento electoral de VOX en las elecciones generales de noviembre de 2019. En particular, se testa el impacto de dos factores organizativos -estatus socioeconómico de los candidatos e implantación territorial-, a través de análisis multivariante. Los resultados señalan la importancia de la esfera organizativa en el rendimiento electoral de la derecha radical en España y apuntan en la dirección de seguir profundizando en el estudio de los factores de la oferta política.

Palabras clave: derecha radical; organización partidista; rendimiento electoral; España.

\begin{abstract}
In recent decades, the growing prominence of the radical right party family of parties in Europe has received considerable academic attention, with one of the main objectives being to study their electoral performance. In this sense, the literature has given a preeminent role to the demand-side factors. The supply factors - which are those that refer to the characteristics of the parties themselves - have received much less attention. Nonetheless, there are reasons to think that, given the limitations of demand-centered explanations, the research agenda on the radical right needs a kind of 'internalist turn'. Or what is the same: a more rigorous and systematized look at the supply-side factors (and especially, on those related to the party organization). The aim of this research is to examine the impact of the organizational dimension
\end{abstract}


on the electoral performance of VOX in November 2019 national elections. In particular, the impact of two organizational factors -socioeconomic status of the candidates and territorial implantation- is tested, through multivariate analysis. The results show the importance of the organizational sphere in the electoral performance of the radical right in Spain and point in the direction of continuing to deepen the study of the supply-side factors.

Keywords: radical right; party organization; electoral performance; Spain.

\section{INTRODUCCIÓN}

Esta investigación tiene como objeto arrojar luz acerca de la siguiente pregunta de investigación: ¿cuál es el impacto de determinados factores relacionados con la organización partidista concretamente, el estatus socioeconómico de los candidatos y la implantación territorial- en el rendimiento electoral de VOX? A partir de una base de datos de elaboración propia y de análisis multivariantes se mostrará que los factores organizativos tienen una efecto importante y positivo en el rendimiento electoral de la derecha radical en España.

El caso español ha sido considerado durante mucho tiempo como una excepción en el contexto europeo debido a la ausencia de una derecha radical institucionalizada (Alonso y Rovira, 2015; Ortiz, Ruiz y González, 2020). Más recientemente, la irrupción electoral de VOX en diferentes arenas ha puesto fin al llamado "excepcionalismo español". Aunque desde el punto de vista de la demanda se ha analizado la base electoral del partido (Ortiz, 2019; Turnbull-Dugarte, 2019), no existen hasta al momento aproximaciones que hayan considerado de forma sistemática los factores de la oferta política -y concretamente, los relacionados con la organización partidista- con relación al rendimiento de VOX. Y es que, considerando los déficits de las explicaciones basadas en la demanda, la agenda de investigación sobre la derecha radical parece necesitar de una suerte de "giro internalista": una mira más sistemática, rigurosa y detallada sobre estos factores y su impacto en el rendimiento electoral.

Considerando lo anterior, la presente investigación se centra en examinar el rendimiento electoral de VOX a nivel provincial en las elecciones generales de noviembre de 2019. Se trata de explorar el efecto de variables como el estatus socioeconómico de los candidatos y el nivel de implantación del partido sobre dicho rendimiento electoral. A su vez, otras variables tradicionalmente consideradas en este estudio serán consideradas como controles.

La investigación está estructurada como sigue. En primer lugar, se hace un repaso crítico por las principales explicaciones dominantes sobre el rendimiento electoral de la derecha radical, que se han basado principalmente en los factores de la demanda. A continuación, y de forma complementaria, se plantea una suerte de giro internalista que permita considerar con mayor detalle también los factores de la oferta (y en especial, los relativos al ámbito de la organización partidista). En este punto, se plantean hipótesis acerca del impacto de determinados factores de la oferta sobre el rendimiento electoral de la derecha radical en España. En la sección tercera se tratan los principales elementos del diseño, mientras que en el apartado cuarto se presentan los resultados. Por último, se sintetizan los hallazgos y se apuntan posibles futuras vías de investigación de cara a implementar una agenda de investigación sobre la derecha radical que considere los factores de la oferta ( $\mathrm{y}$ en concreto, los que tienen que ver con el plano organizativo) de forma sistemática y con vocación comparada. 


\section{MARCO TEÓRICO}

En este apartado se realiza un repaso de la agenda de investigación sobre la derecha radical, que ha estado tradicionalmente dominada por lo que se ha venido a denominar como "perspectiva externalista". Desde un punto de vista crítico, se subrayarán algunos de sus déficits y se sugerirán algunas claves de cara a implementar de forma complementaria una "enfoque internalista".

\subsection{Una mirada crítica a las explicaciones dominantes sobre la derecha radical: ¿más allá de la demanda?}

Desde los años 80, la emergencia de partidos de derecha radical en numerosos sistemas de partidos europeos ha venido acompañada de un interés creciente de la literatura, hasta el punto en que esta familia de partidos ha recibido mucha más atención que otras, aun cuando su influencia real es limitada (Mudde, 2016). En general, la literatura académica ha abordado la irrupción de estos partidos como un fenómeno anómalo que solo puede ser explicado como consecuencia de circunstancias extremas y especiales. Este prisma evoca -cuando no reproduce explícitamente- los principios de buena parte de la literatura sobre el Fascismo Clásico en el periodo de entreguerras. No obstante, los partidos de derecha radical modernos no parecen ser actores ajenos u antagónicos de la democracia liberal, sino más bien reflejos de algunas de sus tensiones inherentes con relación a temas centrales como la identidad, la representación o el multiculturalismo (Mudde, 2010). Las constantes analogías con el Fascismo Clásico no parecen ser muy adecuadas si se tienen en cuenta las notables diferencias entre el contexto del periodo de entreguerras y el panorama Europeo (Art, 2013).

Sea como fuere, este paradigma hegemónico está anclado en la idea general de crisis. Dicho de otra manera, la irrupción de la derecha radical es concebida como una consecuencia directa de crisis económicas, políticas o sociales, en sentido amplio. Este marco ha sido cíclicamente reconceptualizado, siendo en la actualidad la denominación de la tesis de los "perdedores de la globalización" una de las más extendidas (Mudde, 2007). Este enfoque combina las aportaciones de las teorías de la desintegración social y de la privación relativa, de forma que la derecha radical es entendida como una reacción alienada de resentimiento de aquellos grupos que han visto mermar su estatus socio-económico como consecuencia de los procesos de modernización y globalización capitalista (Rydgren, 2007). Bell sintetiza perfectamente esta visión, al describir a la derecha radical como: "la política de la frustración, la agria impotencia de quienes son incapaces de comprender, y menos aún de mandar, en la compleja sociedad de masas actual" (2002: 42). 
Figura 1. Resultados electorales de partidos de derecha radical en Europa en elecciones nacionales (1980-2020).

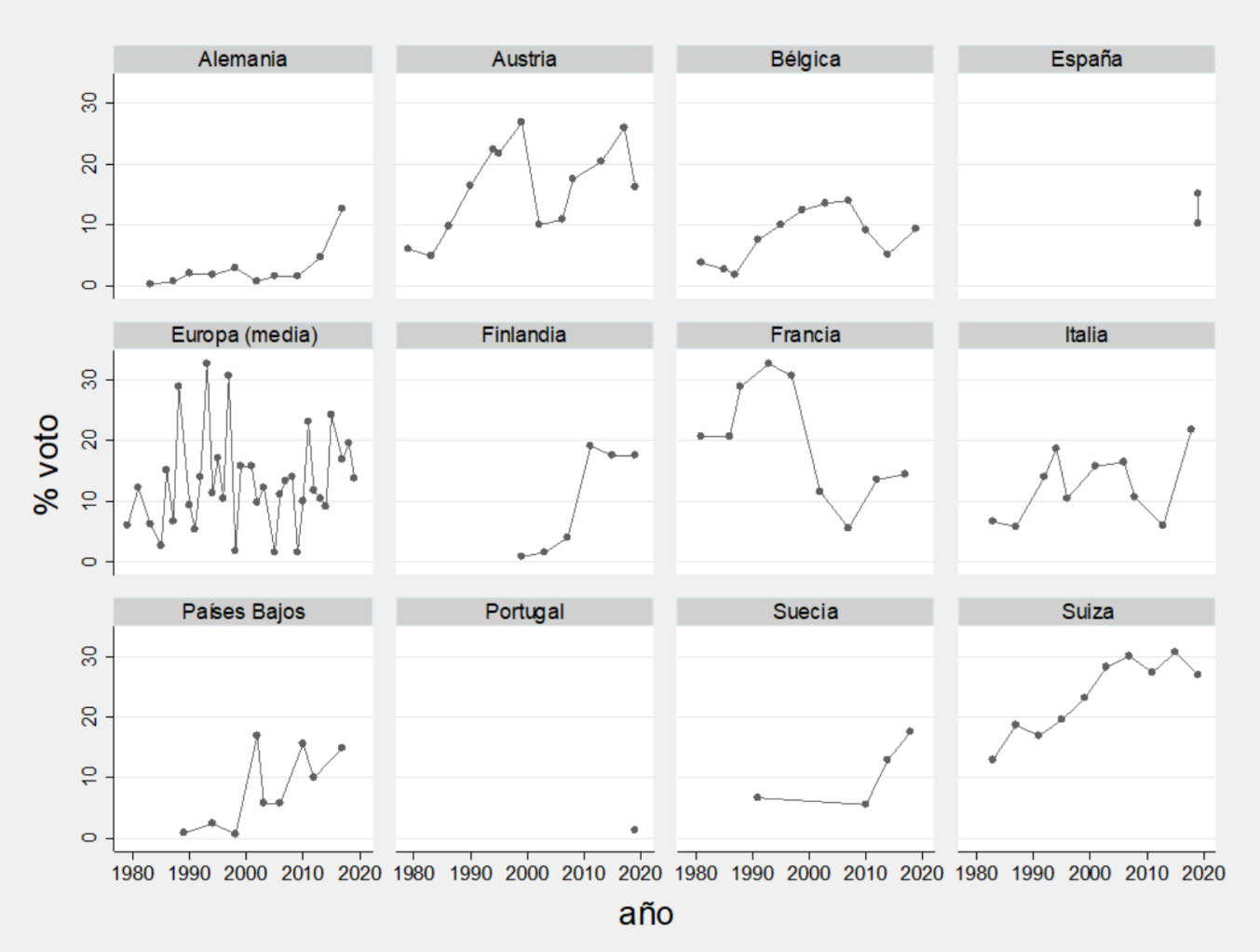

Fuente: elaboración propia a partir de Döring y Manow (2020)

Con respecto a este enfoque hegemónico, es posible identificar lo que Goodwin (2006) ha denominado como el sesgo externalista, que consiste en la excesiva atención sobre los factores de la demanda. Los factores de la demanda son aquellos que se refieren a las actitudes, preferencias y orientaciones de la sociedad y el electorado, así como a los procesos estructurales que los condicionan (Norris, 2005; Rydgren, 2007). El énfasis sobre los factores de la demanda puede ser interpretado en última instancia como reflejo del dominio de la estructura sobre la agencia en las últimas décadas en las Ciencias Sociales. Una lectura extrema de esta "perspectiva externalista" llevaría a fijarse solamente en los votantes, más que en los partidos. Mas, ¿no deberían ser los partidos el objeto de estudio primario de la agenda de investigación sobre la derecha radical? Al fin y al cabo, los interrogantes centrales de la literatura van en la misma dirección: indagar en por qué la derecha radical tiene éxito (o no) y cuáles son sus efectos. Indudablemente, los factores de la demanda pueden ayudar a arrojar luz sobre los procesos y tendencias sociales que están detrás de este fenómeno. No obstante, las explicaciones centradas en la demanda son solo una parte del rompecabezas y fallan a la hora de explicar el verdadero rasgo que caracteriza a la derecha radical en Europa: la variabilidad geográfica y temporal de su desempeño electoral (o, dicho de otra manera, la alternancia de éxitos y fracasos tanto en perspectiva within-country como cross-country) (ver figura 1). las explicaciones basadas en la demanda son incapaces de abordar por sí mismas esta variabilidad: ¿por qué la derecha radical ha fracasado en contextos donde, de acuerdo con las explicaciones de la demanda, debería haberlo hecho bien?, ¿cómo se explica el éxito en escenarios 
donde no había a priori factores de la demanda propicios? En este sentido, son numerosos los trabajos que han venido apuntando la necesidad de superar el reduccionismo y las debilidades de ciertas expresiones del "enfoque externalista" (Acha, 2017; Art, 2011; Arzheimer, 2009; Norris, 2005).

\subsection{Hacia un giro internalista: derecha radical y organización partidista}

Asumiendo las limitaciones de la "perspectiva externalista" tratada en el epígrafe anterior, parece necesario un giro en la materia. Aunque se supone que existe un consenso sobre la necesidad de considerar conjuntamente tanto factores de la demanda como de la oferta, lo cierto es que los factores de la oferta -que son aquellos que se refieren expresamente a los partidos en sí mismos y a su entorno político (Rydgren, 2007)- son todavía mucho menos relevantes en el conjunto de la literatura. Una suerte de giro en el foco y una mirada más certera hacia los factores de la oferta, que se ha venido a denominar como la "perspectiva internalista", podría contribuir a enriquecer el campo de estudio, de forma que estos partidos no sean considerados como "desdichadas víctimas de su entorno económico o demográfico, sino como artífices activos de su propio destino" (Berman, 1997). Esto implica aceptar que los partidos de derecha radical tienen cierto margen de maniobra y que, en última instancia, la agencia (y no solo la estructura) importa. Como apunta Mudde: "es necesario poner a los partidos en el centro del estudio sobre el fenómeno" (2007: 295).

Dentro de esta "perspectiva internalista", una línea de investigación todavía infradesarrollada es la que tiene que ver con la esfera organizativa. La construcción de organizaciones sólidas y eficientes es clave para que la derecha radical pueda lograr estabilidad y persistencia electoral (Art, 2011; Carter, 2005; Mudde, 2007). En términos generales, existe una sorprendente laguna en torno a la dimensión organizativa si se compara con la gran atención que han recibido otros temas como las orientaciones de los votantes o la ideología de estos partidos

Siguiendo a de Lange y Art (2011), parece que más que la organización per se, el factor clave es la forma en que los partidos se institucionalizan. La institucionalización organizativa puede ser definida como "el proceso por el cual una organización, de ser un medio para un fin, se convierte en un fin en sí misma" (Gunther y Hopkin, 2002: 196). Dada la complejidad de esta idea, parece necesario concebirla más bien como un concepto multidimensional. De acuerdo con Janda (1980), es posible distinguir dos dimensiones de la institucionalización partidista: por un lado, una dimensión interna que se refiere a la configuración interna del partido y alude a la existencia de rutinas de comportamiento y a la "infusión de valores" (es decir, cómo los militantes adquieren identificación con el partido); por otro, una dimensión externa que se refiere a las relaciones del partido con la sociedad y su entorno institucional y político.

¿Cómo afecta concretamente la institucionalización organizativa al rendimiento electoral de los partidos de derecha radical? Casi todos los partidos de derecha radical que obtienen apoyo y representación por primera vez están pobremente desarrollados desde el punto de vista organizativa. Así, en el momento de su irrupción, los partidos de derecha radical aprovechan factores como el issue ownership, es decir, la forma en que el partido aprovecha su credibilidad en determinados issues. En el caso de VOX, esto se aprecia antes su irrupción en la arena autonómica andaluza en diciembre de 2018, cuando el partido aprovechó el contexto de polarización sobre la cuestión territorial para hacer valer sus planteamientos centralistas (Ortiz, 2019). Sin embargo, conseguir sostenibilidad y persistencia es un reto mucho más difícil que simplemente "irrumpir" 
por primera vez. Para esta empresa, los partidos necesitan desarrollar un liderazgo sólido, desarrollar mecanismos organizativos eficientes para gestionar las tensiones, reclutar candidatos competentes y creíbles, contratar personal profesionalizado y extenderse por el territorio, entre otras cuestiones. Cuando los partidos consiguen desarrollar organizaciones fuertes, coordinadas y homogéneamente distribuidas por todo el territorio, más probable es que los votantes les otorguen credibilidad y los vean como opciones serias. Por el contrario, cuando los partidos son percibidos como caóticos, desorganizados o incoherentes, más probable es que no reciban apoyo. Por todo ello, la institucionalización organizativa juega un papel clave en la estabilización del partido a medio y largo plazo en la arena electoral (Art, 2008).

Un aspecto relevante dentro de la dimensión interna de la institucionalización organizativa de la derecha radical es aquel que tiene que ver con el capital humano. Tradicionalmente, los partidos de derecha radical que han estado estigmatizados en sus respectivos sistemas de partidos han tenido dificultades para reclutar militantes y candidatos competentes y con buena valoración pública (de Lange y Art, 2011). En su brillante trabajo comparativo, Art (2011) ha demostrado a través de entrevistas en profundidad en varios países de Europa cómo los partidos de derecha radical marginales tienen una pauta que se repite: cuentan con una base militante y de candidatos con orientación extremista y perfil socioeconómico bajo. La existencia de candidatos y activistas con este perfil poco valorado socialmente refuerza la imagen de "outsiders" de estos partidos y redunda negativamente en su rendimiento electoral. Al contrario, la presencia de candidatos con considerable estatus socioeconómico tiene un efecto positivo en el rendimiento electoral del partido por dos vías: por un lado, su posición acomodada y su reconocimiento social les permite actuar de altavoces del partido (ya sea expandiendo en su entorno las ideas del mismo o haciendo que personas cercanas se afilien); por otro, con candidatos con alto estatus el partido gana credibilidad y reputación (algo especialmente relevante para la derecha radical, que es a menudo vista con recelo para buena parte de la sociedad). En suma, se trata de un factor organizativo casi ignorado por la literatura, pero del que los pocos trabajos empíricos que lo han abordado han señalado su importancia (Erlingsson, Loxbo y Öhrvall, 2012; Loxbo y Bolin, 2016). De esta forma, es posible trazar un nexo causal entre el estatus socioeconómico de los candidatos y el rendimiento electoral de la derecha radical. Trasladando esta idea al caso de estudio (VOX y su rendimiento electoral en las elecciones generales de 2019), la hipótesis 1 plantea que:

H1: a mayor nivel de estatus socioeconómico de los candidatos, se espera mejor rendimiento electoral de VOX en cada provincia.

Otro aspecto relevante de la institucionalización organizativa de los partidos políticos, en general, y de la derecha radical, en particular, es el de la implantación territorial. La presencia del partido a lo largo del territorio es un buen indicador de su fortaleza organizativa y remite, en última instancia, a la existencia de cierta complejidad organizativa, de dinámicas de coordinación y de una base suficiente de militancia. De este modo, estudios anteriores han señalado la importancia que tiene la presencia de la derecha radical en la esfera local, pues es allí donde se desarrolla en mayor medida la socialización y el reclutamiento de sus militantes y la expansión de sus ideas (Dinas, Georgiadou, Konstantinidi y Rori, 2016; Werkmann y Gherghina, 2016). El anclaje organizativo a nivel local permite al partido cimentarse y generar lazos con la sociedad, lo cual redunda positivamente en su rendimiento electoral. Así pues, la hipótesis 2 se plantea de la siguiente manera:

H2: a mayor implantación territorial de VOX a nivel provincial, se espera mejor rendimiento electoral del partido en dicha provincia. 


\section{DISEÑO METODOLÓGICO}

Este estudio se centra en examinar el impacto de ciertos factores relacionados con la organización partidista -estatus socioeconómico de los candidatos e implantación territorial- en el rendimiento electoral de un partido de derecha radical (VOX). La literatura existente hasta el momento califica de forma unánime a VOX como parte de la familia partidista de la derecha radical, en la medida en que entre sus planteamientos es posible identificar principios como el nativismo o el autoritarismo, que son los que conforman el núcleo ideológico mínimo de este tipo de partidos (Ortiz, 2019; Ortiz et al., 2020; Ramos-González y Ortiz, 2021).

La elección de VOX como objeto de estudio responde a varios motivos. Tras la dictadura franquista, España fue durante décadas considerada una excepción en el contexto europeo ya que, a pesar de contar en determinados momentos con condiciones a priori favorables para el crecimiento de la derecha radical, ninguna fuerza de este tipo fue capaz de institucionalizarse. Sin embargo, el llamado paradigma de la excepcionalidad española -que, por otra parte, descansaba en un conjunto de postulados excesivamente mecanicistas y acríticos con relación a los factores que determinan el rendimiento de la derecha radical, como han señalado Ortiz, Ruiz y González (2020)-, saltó por los aires con la entrada de VOX en el Parlamento andaluz en diciembre de 2018 y su posterior aterrizaje en la arena nacional, autonómica, municipal y europea. En poco tiempo, y por el momento, VOX ha sido capaz de hacerse hueco en el panorama político español al punto de ser la tercera fuerza en el Parlamento.

En suma, VOX es un partido de reciente irrupción que se encuentra actualmente en pleno proceso de institucionalización organizativa. En este sentido, indagar en el papel de factores de la oferta relacionados con la organización partidista resulta de gran interés, pues trabajos previos han señalado su influencia en la sostenibilidad electoral a medio y largo plazo (Art, 2011; Loxbo y Bolin, 2016; Mudde, 2007; Ortiz, Ruiz, y González, 2021).

La variable dependiente de esta investigación es el rendimiento electoral, que se ha operacionalizado a partir del índice propuesto por Gordin (2001). Este índice de éxito electoral se calcula dividiendo el porcentaje de votos del partido en cuestión entre el porcentaje de votos del partido más votado. Así pues, se ha calculado para cada una de las 52 provincias la siguiente fórmula:

\section{índice éxito electoral $=\frac{\% \text { votos VOX }}{\% \text { votos partido más votado }}$}

El índice de éxito electoral oscila entre 0 (indicando un nulo resultado por parte de VOX) y 1 (indicando que VOX es el partido más votado en esa provincia). Este índice tiene la ventaja de ser intuitivo y capturar la naturaleza de la competición electoral en cada provincia, abordando el rendimiento del partido en términos relativos (es decir, con relación al partido más votado en cada circunscripción). Otras operacionalizaciones alternativas -como el uso del porcentaje de votos del partido o de los escaños obtenidos en cada provincia- tienen el inconveniente de no ser tan útiles de cara a la comparación, habida cuenta de las diferentes características con del sistema electoral en cada provincia. En definitiva, la solución adoptada ofrece una medida estandarizada y comparable entre las diferentes unidades territoriales. 
Las principales variables independientes de la investigación son el estatus socioeconómico de los candidatos y la implantación territorial del partido. Para la operacionalización del estatus socioeconómico de los candidatos se ha llevado a cabo un doble proceso. En primer lugar, se han recopilado las listas oficiales de candidatos de VOX para las elecciones generales de noviembre de 2019 a través del Ministerio del Interior (2020). En total, el partido presentó 349 candidatos. A continuación, se ha realizado una búsqueda de las profesiones de los candidatos por diferentes vías: ya sea por fuentes oficiales (Parlamento, parlamentos regionales, información oficial proporcionada por el partido), prensa o a través de una exploración informal en la web. Hay que tener en cuenta la creciente extensión de las redes sociales y de portales de búsqueda de empleo, que hace que sea común que los candidatos exhiban de forma pública su profesión y/o cualificación. Este proceso ha sido muy fructífero, en la medida en que se ha encontrado la profesión para 267 candidatos. Es decir, se ha encontrado la profesión para el 76\% de los candidatos del partido.

En una segunda etapa, se ha procedido a codificar las profesiones de acuerdo con la clasificación internacional ISCO (International Standard Classification of Occupations) ${ }^{1}$. Se trata de una clasificación que agrupa las profesiones de acuerdo con el nivel de cualificación necesario para desempeñarlas. Es por eso que la profesión puede ser considerada un buen indicador del estatus socioeconómico. Por último, se ha construido una escala propia a partir de la codificación ISCO, en la línea de trabajos previos similares (Art, 2011; Loxbo y Bolin, 2016). Así, el grupo ISCO 9 pertenecería al nivel 1; los grupos ISCO 4 al 8 al nivel 2; el grupo ISCO 3 al nivel 3 y los grupos ISCO 1 y 2 al nivel 4 . Así pues, la escala oscilaría entre 1 y 4 , indicando de menor a mayor nivel de estatus socioeconómico. Por último, una vez se tienen todos los candidatos enmarcados en la escala de estatus socioeconómica, se ha calculado la media para cada provincia. En conclusión, la búsqueda, elaboración y operacionalización de la variable de estatus socioeconómico de los candidatos es uno de los valores añadidos de esta investigación.

La variable de implantación territorial se ha construido utilizando de referencia las elecciones municipales de mayo de 2019. Se ha partido de la base de que la presencia de concejales electos de VOX es un buen indicador de la presencia del partido en cada municipio (y, por ende, también en la provincia). La existencia de concejales remite, en última instancia, a la presencia de una mínima infraestructura organizativa del partido. Asimismo, la cercanía entre ambos comicios (mayo y noviembre de 2019), hace que las elecciones municipales sean un buen proxy de la expansión organizativa del partido. Esta variable se ha operacionalizado calculando el número de concejales electos de VOX para todos los municipios de cada provincia, y dividiendo ese número entre el total de concejales elegibles en dicha provincia. De forma resumida, la fórmula del cálculo es la siguiente: [( $\mathrm{n}^{\circ}$ concejales VOX en provincia/no concejales totales de la provincia)*100]. El resultado arroja un índice de implantación territorial de VOX en cada provincia que permite testar la hipótesis nํㅡ.

Asimismo, se han considerado otras variables del lado de la demanda para controlar factores que han sido comúnmente señalados como importantes para el rendimiento electoral de la derecha radical: desempleo e inmigración. Tal y como se ha revisado en la primera parte del marco teórico, la literatura ha tendido a conectar los escenarios de deprivación económica con el auge de la derecha radical (Arzheimer, 2018; Givens, 2005; Rydgren, 2007). En este este sentido, el marco de los “perdedores de la globalización" plantea que, en la medida en que existan grupos sociales que tienen una posición socioeconómica frágil (o bien la hayan visto mermada), más fácil es que orienten su apoyo a opciones como la derecha radical. Considerando todo esto, se ha operacionalizado la 
variable desempleo tomando la media de la tasa de paro en cada provincia durante el año anterior a las elecciones de noviembre de 2019 (INE, 2021a).El impacto que tiene esta variable en el apoyo electoral no es instantáneo, sino que está sometido a cierta evaluación retrospectiva: es por eso que se ha tomado un periodo de tiempo amplio con anterioridad a las elecciones objeto de estudio.

Por otro lado, la inmigración es clave para la derecha radical, siendo los postulados antiinmigración uno de los pilares principales de su oferta programática ${ }^{2}$. La cuestión de la inmigración es estrechamente conectada con la tesis de los "perdedores de la globalización" (examinada por medio del desempleo), ya que, en la medida en que las condiciones socioeconómicas sean más adversas, más probable es que se culpabilice a los "otros" (en este caso, a los inmigrantes), en virtud de las teorías sobre los conflictos intergrupales y la competencia étnica (Arzheimer, 2009). Esta variable se ha operacionalizado a través del porcentaje de población extranjera en cada provincia a fecha de 1 enero de 2020, según el INE (2021).

Además, se ha introducido una cuarta variable de control del lado de la oferta que guarda relación con la llamada estructura de oportunidades políticas. Tarrow define la estructura de oportunidades políticas como "las dimensiones del entorno político que son consistentes, aunque no necesariamente formales o permanentes, y que proporcionan incentivos para emprender la acción colectiva afectando las expectativas de éxito y fracaso" (1994: 85). La variable usada tiene que ver con el impacto de las reivindicaciones nacionalistas periféricas o regionalistas en cada una de las provincias. En tanto que VOX se ha conectado ideológicamente con un ultranacionalismo español de corte centralista y esencialista, se espera que el rendimiento electoral del partido sea menor en aquellos territorios donde existan reivindicaciones nacionalistas o regionalistas diferentes al españolismo. Al contrario, allí donde no haya este tipo de identidades canalizadas políticamente, se hipotetiza que el rendimiento de la derecha radical será mejor. Para operacionalizar esta variable, se ha calculado para cada provincia el porcentaje de votos de partidos categorizados como nacionalistas o regionalistas en las dos elecciones anteriores (2016 y abril 2029).

Por último, respecto al tipo de análisis, se va a utilizar la regresión lineal OLS de cara a examinar el impacto de las variables independientes sobre el rendimiento electoral de la derecha radical y en conjunción con los diferentes controles.

\section{RESULTADOS}

A continuación, se presentan los análisis multivariantes. En concreto, la tabla 1 muestra tres modelos de regresión OLS para explicar el rendimiento electoral de VOX en las provincias españolas en las elecciones generales de 2019. En el primer modelo se testan las principales variables explicativas del estudio: el estatus socioeconómico de los candidatos y la implantación territorial. En el segundo se introducen las variables de control: desempleo, inmigración y movilización territorial subestatal. El tercer modelo es el modelo completo, donde se trata de analizar conjuntamente el poder explicativo de todas las variables. Para facilitar la comparación de las distintas variables se muestran los coeficientes beta estandarizados.

El primer modelo tiene en consideración el estatus de los candidatos y la implantación territorial. En cuanto a la primera variable explicativa, el coeficiente estandarizado indica una relación negativa con respecto al rendimiento electoral. Aunque este signo no se corresponde con la dirección esperada en la hipótesis de partida, el valor obtenido es muy cercano a cero. Este resultado se puede 
explicar a partir de la escasa variabilidad que presenta la variable estatus, fruto de la homogeneidad interna que caracteriza a las candidaturas de VOX. Con respecto a la implantación territorial, el efecto se muestra positivo y significativo, apuntando una vinculación favorable al rendimiento electoral cuanto mayor es dicha implantación. El valor de su coeficiente estandarizado es de 0,530, por lo que, manteniendo constante la otra variable, el partido vería aumentado rendimiento electoral más de medio punto por cada punto de incremento en la implantación. Este modelo de sólo dos variables alcanza una capacidad explicativa considerable, presentando un valor de $\mathrm{R}^{2}$ ajustado de 0,25 .

\begin{tabular}{|c|c|c|c|}
\hline Estatus candidatos & $\begin{array}{c}-0.012 \\
(0.0649)\end{array}$ & & $\begin{array}{c}0.002 \\
(0.0408)\end{array}$ \\
\hline Implantación territorial & $\begin{array}{l}0.530^{* * *} \\
(0.0260)\end{array}$ & & $\begin{array}{l}0.299^{* * *} \\
(0.0177)\end{array}$ \\
\hline Inmigración & & $\begin{array}{c}0.471^{* * *} \\
(0.00365)\end{array}$ & $\begin{array}{c}0.424^{* * * *} \\
(0.00333)\end{array}$ \\
\hline Desempleo & & $\begin{array}{c}0.333^{* * *} \\
(0.00366)\end{array}$ & $\begin{array}{c}0.221^{*} \\
(0.00358)\end{array}$ \\
\hline Movilización territorial & & $\begin{array}{l}-0.676^{* * *} \\
(0.00138)\end{array}$ & $\begin{array}{l}-0.639^{* * *} \\
(0.00126)\end{array}$ \\
\hline $\begin{array}{l}N \\
\text { adj. } R^{2}\end{array}$ & $\begin{array}{c}52 \\
0.252\end{array}$ & $\begin{array}{c}52 \\
0.645\end{array}$ & $\begin{array}{c}52 \\
0.712\end{array}$ \\
\hline
\end{tabular}

Coeficientes beta estandarizados; errores estándar entre paréntesis $* p<0.05,{ }^{* *} p<0.01,{ }^{* * *} p<0.001$

Fuente: elaboración propia

En el modelo 2 se han introducido únicamente las variables de control seleccionadas con el objetivo de comprobar su impacto en la variable dependiente. La primera de ellas es la inmigración, que se muestra significativa con carácter positivo, lo que apunta a un mejor rendimiento electoral de la formación a medida que se incrementa el peso de la población migrante en la provincia (en 0,471 puntos por cada punto de incremento en la variable dependiente). Asimismo, el desempleo también ofrece un coeficiente significativo de signo positivo, aunque con un valor beta menor al que presenta la inmigración. Por último, se ha introducido como variable de control la presencia de partidos nacionalistas a nivel subestatal. Esta se muestra significativa, al igual que las anteriores, aunque con signo negativo. En este sentido, a menor presencia de partidos de tales características en una región, mayor éxito electoral podrá esperarse de VOX. De las variables consideradas en este segundo modelo, esta es la que presenta un mayor coeficiente beta $(0,676)$. Esto va en la línea de estudios anteriores que han señalado la importancia de la cuestión territorial en la irrupción 
electoral de VOX (Ortiz, 2019; Turnbull-Dugarte, 2019). El modelo 2 presenta una alta capacidad explicativa, alcanzando un valor de $\mathrm{R}^{2}$ ajustado de 0,64 .

En el tercer modelo, los resultados apuntan a una relativa robustez de las variables explicativas, que muestran una gran similitud entre los coeficientes estandarizados de este modelo y de los anteriores. La variable de estatus socioeconómico de los candidatos es positiva, aunque muy cercana a 0 y sin ser significativa, mientras que el resto de las variables mantienen su signo y su nivel de significancia. La implantación territorial afectaría de forma positiva al rendimiento electoral del partido. Es decir, a mayor implantación territorial, mayor éxito electoral (en la línea con lo planteado en la hipótesis 2). De esta forma, si se mantienen el resto de las variables constantes, el éxito electoral aumentará 0,29 puntos en desviaciones típicas por cada punto adicional de implantación territorial. Del mismo modo, las variables inmigración y desempleo tienen un efecto positivo y significativo, al igual que en el segundo modelo. A mayores niveles de desempleo e inmigración, el éxito electoral de Vox es también mayor (en 0,42 y en 0,22 desviaciones típicas, respectivamente). Por último, la movilización subestatal sigue siendo significativa en la dirección esperada y el coeficiente de mayor tamaño. Esto implica que un mayor éxito de Vox en una circunscripción electoral está relacionado con una baja movilización de partidos no estatales (-0,63 puntos). Esto apunta a que VOX tiene mejor rendimiento en territorios donde no hay reivindicaciones nacionalistas periféricas o regionalistas (o, dicho de otro modo, que la derecha radical tiene mejor resultados allí donde el nacionalismo español tiene preeminencia). Finalmente, el modelo consigue una alta bondad de ajuste con un $\mathrm{R}^{2}$ ajustado de 0,71 .

En vista del modelo 3, no es posible aceptar ni refutar de forma concluyente la hipótesis 1 que planteaba un efecto positivo del estatus socioeconómico de los candidatos en el rendimiento electoral del partido. En este punto, cabría preguntarse acerca de un posible efecto conjunto entre las dos principales variables independientes: estatus candidatos e implantación territorial. Para ello, se ha replicado el modelo 3, incluyendo una interacción entre estatus candidatos e implantación territorial. Este modelo 4 puede consultarse en el apéndice en la tabla A2.

Dado que es difícil interpretar la interacción a través de su coeficiente o significación, se ha optado por representarla de forma gráfica siguiendo las sugerencias de Kam y Franzese (2009). La figura 2 representa los efectos marginales de estatus socio-económico de los candidatos condicionado a implantación territorial. Dicha figura muestra cómo la interacción pasa a ser significativa cuando la implantación territorial alcanza un valor igual o superior al 2. Es decir, cuando existe un determinado nivel de implantación territorial en una provincia, el aumento del nivel de estatus socioeconómico de los candidatos influye positivamente en el éxito en el rendimiento electoral del partido. El nivel de estatus socioeconómico de los candidatos parece estar, por tanto, condicionado de forma significativa a la implantación organizativa del partido. 
Figura 2. Efectos marginales de estatus socioeconómico de candidatos condicionado a implantación territorial (intervalos de confianza 95\%)

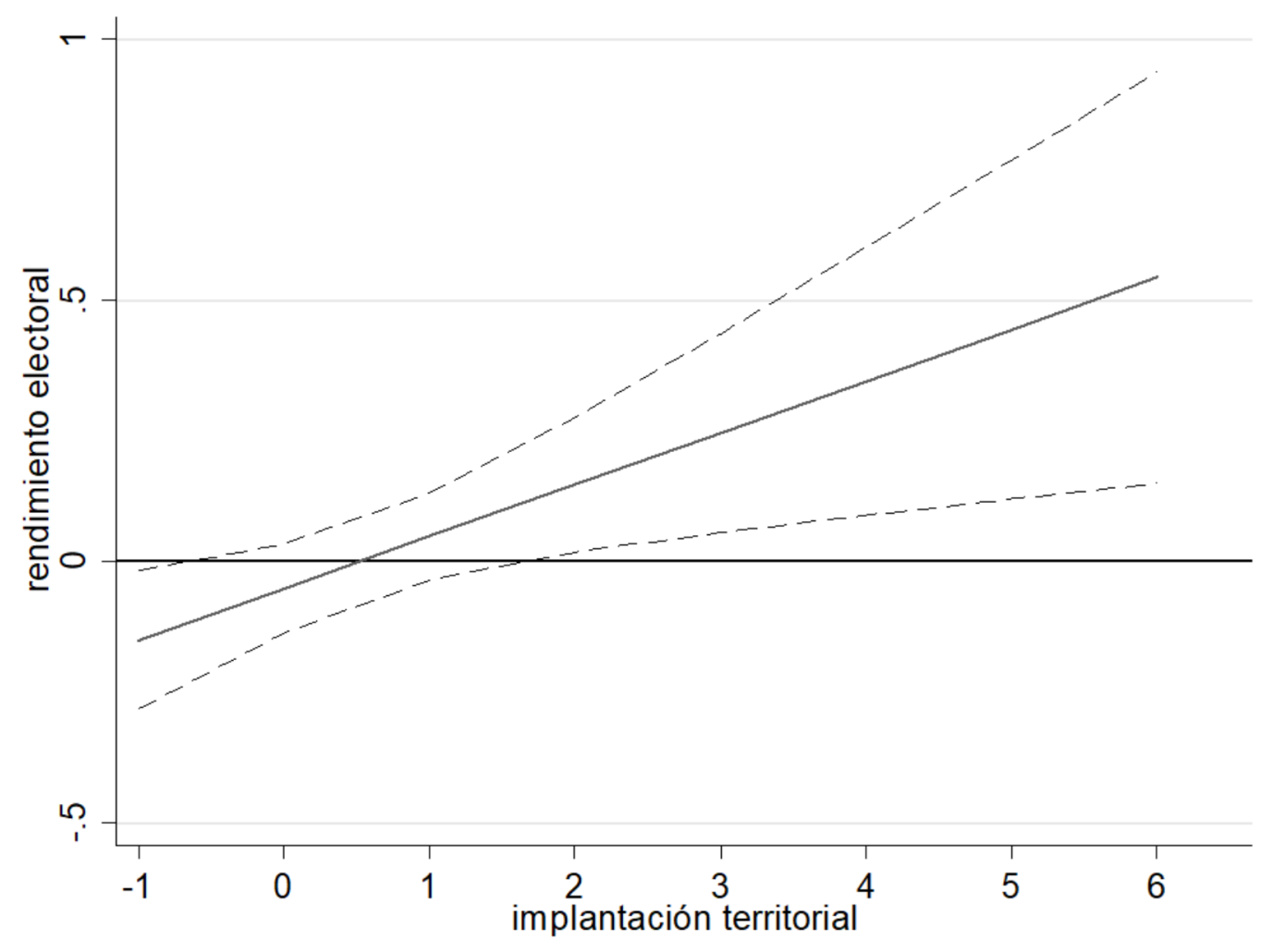

Fuente: elaboración propia a partir de tabla A2. El resto de las variables se mantienen en sus valores medios.

En suma, los resultados apuntan de manera exploratoria que existe un efecto positivo de los factores de la oferta relacionados con la organización partidista en el rendimiento electoral de la derecha radical en España. Asimismo, el resto de los factores considerados (desde la demanda: inmigración y desempleo; desde la oferta y la estructura de oportunidades políticas: la movilización política territorial) parecen tener un papel importante en conjunción con estos factores organizativos. En definitiva, todo parece apuntar a la necesidad de considerar conjuntamente tanto los factores de la oferta como de la demanda en el abordaje de la derecha radical

\section{DISCUSIÓN Y CONCLUSIONES}

Desde los años 80, el creciente protagonismo de los partidos de derecha radical en Europa ha venido acompañado de un gran interés por examinar los factores que pueden explicar su rendimiento electoral. En este sentido, las explicaciones basadas en los factores de la demanda han sido, en términos comparados, utilizadas en mayor medida que aquellas centradas en la oferta (a pesar de las llamadas a combinar ambos tipos de factores). Respecto al caso de España, la reciente irrupción electoral de VOX ha puesto fin a décadas de marginalización de esta familia de partidos. Esta investigación se ha ocupado de abordar en qué medida el rendimiento electoral de VOX en las 
elecciones generales de noviembre de 2019 puede venir explicado por determinados factores de la oferta relacionados con la organización partidista.

Los análisis multivariantes revelan que los dos factores de la organización partidista considerados -estatus socioeconómico de los candidatos e implantación territorial del partidotienen un efecto positivo en el rendimiento electoral de VOX a nivel provincial, controlando por otros factores del lado de la demanda como desempleo e inmigración. Aunque la variable de estatus socioeconómico de los candidatos no arroja significación estadística al ser examinada en solitario, la interacción entre esta variable e implantación territorial sí resulta significativa. De este modo, el estatus de los candidatos parecer impactar positivamente y de forma significativa allí donde se alcanza cierto nivel de implantación organizativa del partido. Tal y como han apuntado estudios previos (Art, 2011; Loxbo y Bolin, 2016), el reclutamiento de candidatos con buena posición económica y preparación académica contribuye a que la derecha radical deje a un lado la tradicional imagen de extremismo y estigmatización que la ha lastrado históricamente, de forma que gane legitimidad y respetabilidad ante la sociedad. A su vez, candidatos con mayor estatus tienen mejores herramientas cognitivas y de capital social, económico y cultural de cara a hacer campaña por el partido y atraer apoyos.

Por otro lado, se ha visto cómo el nivel de presencia organizativa del partido a nivel local es un buen predictor de su rendimiento electoral a nivel provincial. Allí dónde VOX ha sido capaz de anclarse organizativamente en el territorio, se observa que es capaz de obtener mejores resultados. Esta cuestión, señalada por estudios previos (Carter, 2005; Mudde, 2007), afecta no solo a la derecha radical, sino en general a todos los partidos políticos. Lo que ocurre es que para la derecha radical la empresa de extenderse organizativamente es más complicada si cabe, habida cuenta del rechazo que genera entre amplios sectores de la sociedad. Más aún, el caso español muestra las dificultades que enfrenta la derecha radical para establecerse organizativamente en determinados territorios (particularmente, allí donde existen reivindicaciones nacionalistas periféricas).

En suma, los hallazgos subrayan la importancia de los factores de la oferta, y en concreto, de aquellos que tienen que ver con el ámbito organizativo. La institucionalización organizativa parece ser crucial no solo para el rendimiento inmediato, sino también para la sostenibilidad electoral de la derecha radical (Arter y Kestilä-Kekkonen, 2014; Bolleyer, 2013; de Lange y Art, 2011).

Esta investigación tiene un carácter exploratorio y su principal objetivo es el de contribuir al debate sobre el papel de los factores de la oferta política en el rendimiento electoral de la derecha radical. El valor añadido de este enfoque reside en la selección y operacionalización de variables poco tratadas con anterioridad, como son el estatus socioeconómico de los candidatos y la implantación territorial. De esta forma, se ha utilizado una base de datos de inédita de elaboración propia. Al mismo tiempo, la aproximación al fenómeno resulta ser bastante parsimoniosa en la medida en que se es capaz de explicar una parte considerable del fenómeno haciendo uso de un modelo estadístico sencillo sin muchos predictores. Asimismo, se subraya la importancia de estudiar el rendimiento electoral de la derecha radical en contextos sub-nacionales, tal y como viene reivindicando desde hace tiempo parte de la literatura (Kestilä y Söderlund, 2007). En definitiva, esta investigación apunta en la dirección de adoptar una 'perspectiva internalista' que considere de forma sistemática los factores de la oferta en conjunción con los de la demanda de cara a fortalecer la agenda de investigación sobre la derecha radical. Se trata, al fin y al cabo, de partir de un prisma 'party-centric' que coloque a los propios partidos en el centro de estudio (Zulianello, 2019).

Ahora bien, este estudio no está exento de limitaciones. Principalmente, lo limitado de la muestra hacer que los hallazgos no sean generalizables a otros contextos. Los resultados, eso sí, sí tienen 
validez en lo concerniente al caso español. También cabe considerar la presencia de otras variables no consideradas por los modelos y que hayan podido impactar en el rendimiento electoral de VOX a nivel provincial. De nuevo, el bajo número de casos disponibles $(\mathrm{N}=52)$ ha hecho que no fuera posible considerar un número demasiado elevado de predictores.

Por último, y en cuanto a posibles futuras vías de investigación, sería interesante combinar esta aproximación cuantitativa con otros enfoques cualitativos que analicen en profundidad cómo operan factores como el estatus de los candidatos y la implantación organizativa y cuál es su vínculo causal con los resultados del partido. Asimismo, se debe avanzar hacia análisis cross-country que examinen de forma sistemática y comparativa el papel de los factores organizativos de la derecha radical en su desempeño electoral.

\section{Bibliografía}

ACHA, Beatriz (2017) "La relación de la crisis económica con el ascenso de los partidos de extrema derecha: el caso de las elecciones europeas de 2014", Inguruak, 63, 1-18.

ALONSO, Sonia y Cristóbal ROVIRA (2015) "Spain: No Country for the Populist Radical Right?", South European Society and Politics, 20 (1), 21-45. https://doi.org/10.1080/13608746.2014.985448.

ART, David (2008) "The organizational origins of the contemporary radical right. The case of Belgium", Comparative Politics, 40 (4), 421-440. https://doi.org/https://doi.org/10.2307/20434094

ART, David (2011) Inside the Radical Right The Development of Anti-Immigrant Parties in Western Europe, Cambridge: Cambridge University Press.

ART, David (2013) "Why 2013 is not 1933: The radical right in Europe", Current History, 112 (752), 88-93. https://doi.org/10.1525/curh.2013.112.752.88.

ARTER, David y Elina KESTILÄ-KEKKONEN (2014) "Measuring the Extent of Party Institutionalisation: The Case of a Populist Entrepreneur Party", West European Politics, 37 (5), 932-956. https://doi.org/10. 1080/01402382.2014.911486.

ARZHEIMER, Kai (2009) "Contextual factors and the extreme right vote in Western Europe, 1980-2002", American Journal of Political Science, 53 (2), 259-275. https://doi.org/10.1111/j.1540-5907.2009.0 0369.x.

ARZHEIMER, Kai (2018) "Explaining Electoral Support for the Radical Right", en J. Rydgren (ed.) The Oxford Handbook of the Radical Right, Oxford: Oxford University Press, 1-29.

BELL, Daniel (2002) The Radical Right, New York: Doubleday.

BERMAN, Sheri (1997) "The life of the party", Comparative Politics, 30 (1), 101-122.

BOLLEYER, Nicole (2013) New parties in old party systems: Persistence and decline in seventeen democracies, Oxford: Oxford University Press.

CARTER, Elisabeth (2005) The extreme right in Western Europe: success or failure?, Manchester: Manchester University Press.

DE LANGE, Sarah. L. y David ART (2011) "Fortuyn versus Wilders: An Agency-Based Approach to Radical Right Party Building", West European Politics, 34 (6), 1229-1249. https://doi.org/10.1080/0140238 2.2011.616662.

DINAS, Elias, Vassiliki GEORGIADOU, Iannis KONSTANTINIDIS y Lamprini RORI (2016) "From dusk to dawn: Local party organization and party success of right-wing extremism", Party Politics, 22 (1), 80-92. ht tps://doi.org/10.1177/1354068813511381. 
DÖRING, Holger y Philip MANOW (2020) "Parliaments and governments database (ParlGov): Information on parties, elections and cabinets in modern democracies", Recuperado de http://www.parlgov.org/.

ERLINGSSON, Gissur Ó, Karl LOXBO y Richard ÖHRVALL (2012) "Anti-immigrant parties, local presence and electoral success", Local Government Studies, 38 (6), 817-839. https://doi.org/10.1080/03003930.2 $\underline{012.740411 .}$.

FERREIRA, Carles (2019) "Vox como representante de la derecha radical en España: un estudio sobre su ideología", Revista Española de Ciencia Política, 51, 73-98. https://doi.org/10.21308/recp.51.03.

GIVENS, Terri E (2005) Voting Radical Right in Western Europe, New York: Cambridge University Press.

GOODWIN, Matthew J (2006) "The rise and faults of the internalist perspective in extreme right studies", Representation, 42 (4), 347-364. https://doi.org/10.1080/00344890600951924

GORDIN, Jorge P (2001) "The electoral fate of ethnoregionalist parties in Western Europe: A boolean test of extant explanations", Scandinavian Political Studies, 24 (2), 149-170. https://doi.org/10.1111/14 67-9477.00051.

GUNTHER, Richard y Jonathan HOPKIN (2002) "A Crisis of Institutionalization: The Collapse of the UCD in Spain", en R. Gunther, J. R. Montero y J. J. Linz (eds.) Political Parties: Old Concepts and New Challenges, Oxford: Oxford University Press, 191-232.

INE (2021a) Tasas de paro por distintos grupos de edad, sexo y comunidad autónoma Unidades: Tasas. Retrieved from https://www.ine.es/jaxiT3/Tabla.htm?t=4247

INE (2021b) Estadística del Padrón Continuo. Datos provisionales a 1 de enero de 2021. Retrieved from htt ps://www.ine.es/jaxi/Tabla.htm?path=/t20/e245/p04/provi/10/\&file=0ccaa002.px

JANDA, Kenneth (1980) Political Parties: A Cross-National Survey, New York: Free Press.

KAM, Cindy y Robert J. FRANZESE (2009) Modeling and interpreting interactive hypotheses in regression analysis, Ann Arbor: University of Michigan Press.

KESTILÄ, Elina y Peter SÖDERLUND (2007) "Subnational political opportunity structures and the success of the radical right: Evidence from the March 2004 regional elections in France", European Journal of Political Research, 46 (6), 773-796. https://doi.org/10.1111/j.1475-6765.2007.00715.x.

LOXBO, Karl y Niklas BOLIN (2016) "Party organizational development and the electoral performance of the radical right: exploring the role of local candidates in the breakthrough elections of the Sweden democrats 2002-2014", Journal of Elections, Public Opinion and Parties, 26 (2), 170-190. https://do i.org/10.1080/17457289.2016.1145685.

MINISTERIO DEL INTERIOR (2020) Resultados electorales. Retrieved from http://www.infoelectoral.mir.e s/infoelectoral/min/

MUDDE, Cas (2007) Populist Radical Right Parties in Europe, Cambridge: Cambridge University Press.

MUDDE, Cas (2010) "The Populist Radical Right: A Pathological Normalcy", West European Politics, 33 (6), 1167-1186. https://doi.org/10.1080/01402382.2010.508901.

MUDDE, Cas (2016) "The Study of Populist Radical Right Parties: Towards a Fourth Wave", C-Rex Working Paper Series No. 1, Oslo: University of Oslo.

NORRIS, Pippa (2005) "Radical Right. Voters and parties in the electoral market", Cambridge: Cambridge University Press.

ORTIZ, Pablo (2019) "The Electoral Breakthrough of the Radical Right in Spain: Correlates of Electoral Support for VOX in Andalusia (2018)", Genealogy, 3 (4). https://doi.org/doi:10.3390/genealogy3040 072.

ORTIZ, Pablo, Antonia María RUIZ y Manuel Tomás GONZÁLEZ (2020) "El caso español y sus implicaciones para el estudio de la ultraderecha: antecedentes y nuevas estrategias de investigación", Revista de Estudios Políticos, 188, 199-220. https://doi.org/10.18042/cepc/rep.188.07. 
ORTIZ, Pablo, Antonia María RUIZ y Manuel Tomás GONZÁLEZ (2021) The role of organizational institutionalization on electoral sustainability. A comparative analysis of the Spanish far right: Fuerza Nueva and VOX.

RAMOS-GONZÁLEZ, Jorge y Pablo ORTIZ (2021) Radicalism and populism: do they always go hand by hand? A comparative ideological analysis of the radical left and the radical right in Spain.

RODRÍGUEZ, José Luis (1992) La extrema derecha en España: del tardofranquismo a la consolidación de la democracia (1967-1982), Madrid: Universidad Complutense de Madrid.

RYDGREN, Jens (2007) "The Sociology of the Radical Right", Annual Review of Sociology, 33 (1), 241-262. $\underline{\mathrm{h}}$ ttps://doi.org/10.1146/annurev.soc.33.040406.131752.

TARROW, Sidney (1994) Power in Movement, Cambridge: Cambridge University Press.

TURNBULL-DUGARTE, Stuart J (2019) "Explaining the end of Spanish exceptionalism and electoral support for Vox", Research \& Politics, 6 (2), 205316801985168. https://doi.org/10.1177/2053168019851680

WERKMANN, Caroline y Sergiu GHERGHINA (2016) "Organized for Parliament? Explaining the Electoral Success of Radical Right Parties in Post-Communist Europe", Government and Opposition, 53 (3), 461485. https://doi.org/10.1017/gov.2016.38.

ZULIANELLO, Mattia (2019) Anti-system parties: From parliamentary breakthrough to government, London: Routledge. https://doi.org/10.4324/9780429422515.

\section{APÉNDICE}

Tabla A1. Descriptivos de las variables

\begin{tabular}{lccccc}
\hline & $\mathrm{N}$ & Mean & $\mathrm{SD}$ & Min & Max \\
\hline Éxito electoral & 52 & 0.488 & 0.214 & 0.061 & 0.992 \\
Estatus candidatos & 52 & 3.634 & 0.401 & 2 & 4 \\
Implantación territorial & 52 & 4.379 & 1.000 & -0.399 & 6,38 \\
Inmigración & 52 & 9.710 & 5.246 & 2,59 & 21,18 \\
Desempleo & 52 & 14.570 & 5.246 & 6,854 & 26,356 \\
Movilización territorial & 52 & 7.635 & 14.566 & 0 & 50,66 \\
& & & & & \\
\hline
\end{tabular}

Nota: elaboración propia 
Tabla A2. Regresión OLS explicando el rendimiento electoral de VOX en las provincias españolas en las elecciones generales de noviembre 2019 (con interacción de estatus e implantación territorial)

Estatus candidatos

Implantación territorial

Estatus candidatos X Implantación territorial

Inmigración

Desempleo

Movilización territorial

$N$

adj. $R^{2}$
$-0.0910$

$-0.0909^{*}$

$0.0280^{* *}$

$0.0202^{* * *}$

$0.0111^{* *}$

$-0.00997^{* * *}$

Unstandardized beta coefficients. ${ }^{*} p<0.05,{ }^{* *} p<0.01,{ }^{* * *} p<0.001$

Fuente: elaboración propia

Notas

1 La clasificación ISCO distingue 9 grandes grupos: grupo 1 (directores y gerentes); grupo 2 (profesionales científicos e intelectuales); grupo 3 (técnicos y profesionales de nivel medio); grupo 4 (personal de apoyo administrativo); grupo 5 (trabajadores de servicios y vendedores de comercios y mercados); grupo 6 (agricultores y trabajadores cualificados agropecuarios, forestales y pesqueros); grupo 7 (oficiales, operarios y artesanos de artes mecánicas y otros oficios); grupo 8 (operadores de instalaciones y máquinas y ensambladores); grupo 9 (ocupaciones elementales). ISCO no enmarca a los militares dentro de ningún grupo, por lo que se han considerado como perdidos en la base de datos. En el caso de los estudiantes, se han considerado como pertenecientes al grupo 2, mientras que los emprendedores se han incluido en el grupo 3.

2 Si bien ahora loss postulados antiinmigración son más importantes para Vox en comparación al momento de su irrupción, el partido todavía está lejos de otros partidos europeos en esta cuestión. En perspectiva histórica, la derecha radical española ha carecido del cariz abiertamente antiinmigración y racista que sí ha sido (y es) preeminente en otros países (Rodríguez, 1992). 